\title{
SOME INVARIANTS OF GENERIC IMMERSIONS AND THEIR GEOMETRIC APPLICATIONS
}

\author{
BY V. POÉNARU \\ Communicated June 20, 1975
}

1. This is an announcement of some theorems to appear in full detail elsewhere [2].

Let $X, Y$ be smooth manifolds, with $\operatorname{dim} X<\operatorname{dim} Y$ and $f: X \rightarrow Y$ a generic immersion. To $f$ we will attach two numerical invariants $\mu_{2}(f)$ and $\nu_{3}(f)$, which will be described in the next paragraphs; it is conceivable that a more cohomological, characteristic-classes-type approach to these invariants should be possible. Anyway, granted their definition one has the following results:

THEOREM. Let $f: X \rightarrow Y$ be a generic immersion as above. The necessary and sufficient condition for the existence of a smooth embedding $X \stackrel{F}{\rightarrow} Y \times R$ lifting $f$ is that $\mu_{2}(f)=\nu_{3}(f)=0$.

$F$ "lifts $f$ " means that the following diagram is commutative:

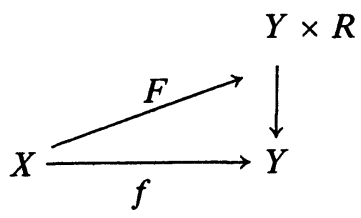

Corollary 1. Suppose that $\pi_{1} X=0$. The necessary and sufficient condition for the existence of a smooth embedding $X \stackrel{G}{\rightarrow} Y \times S_{1}$ lifting $f$ is that $\mu_{2}(f)=\nu_{3}(f)=0$.

The next corollary has some connection with the group $\Theta_{3}$ of Milnor and Kervaire [1]. We consider a smooth homotopy 3-sphere $\Sigma_{3}$ and two points $p_{0}$, $p_{1} \in \Sigma_{3}\left(p_{0} \neq p_{1}\right)$. We consider two small 2-spheres, in $\Sigma_{3}$, of centers $p_{0}$ and $p_{1}: S_{2}^{0}, S_{2}^{1}$. By the Smale-Hirsch immersion theory there is a (generic) regular homotopy:

$$
f \in \operatorname{Imm}_{1}\left(S_{2} \times I,\left(\Sigma_{3}-\left\{p_{0}, p_{1}\right\}\right) \times I\right)
$$

connecting $S_{2}^{0}, S_{2}^{1}$. (The subscript $I$ means that $f$ is level-preserving.)

COROLlARY 2. Let $\Sigma_{3}$ be a smooth homotopy 3-sphere, and $f$ some gener-

AMS (MOS) subject classifications (1970). Primary 22-XX. 
ic regular homotopy as above. If $\mu_{2}(f)=\nu_{3}(f)=0$, then $\Sigma_{3}$ is h-cobordant to $S_{3}$.

2. We shall define now the invariant $\mu_{2}$. Let $M^{i}(f)$ denote the $i$-tuple points of $f$ at the source $X$ :

$$
X \supset M^{2}(f) \supset M^{3}(f) \supset \cdots \supset M^{i}(f) \supset M^{i+1}(f) \supset \cdots
$$

$M^{i}(f)$ is a smooth manifold with singularities. $M^{i+1}(f) \subset M^{i}(f)$ is exactly the singular set.

Now, for an arbitrary set $E$, we consider the $i$ th cartesian power $E^{i}$, the $i$ th symmetric power $S^{i} E \stackrel{P_{i}}{\longleftarrow} E^{i}$, and the two diagonals: $\{x, \ldots, x\}=\operatorname{diag}_{i} E \subset$ $\operatorname{Diag}_{i} E \subset E^{i} . f: X \rightarrow Y$ induces $f^{i}: X^{i} \rightarrow Y^{i}$ and we define the $i$-tuple points at the $X^{i}$, or $S^{i} X$ level, by:

$$
\begin{aligned}
& M_{i}(f)=\left(f^{i}\right)^{-1}\left(\operatorname{diag}_{i} Y\right)-\operatorname{Diag}_{i} X \subset X^{i}, \\
& \hat{M}_{i}(f)=P_{i} M_{i}(f) \subset S^{i} X .
\end{aligned}
$$

Note that $P_{i}: M_{i}(f) \rightarrow \hat{M}_{i}(f)$ is a covering space, and that the spaces involved are smooth (nonsingular) manifolds. Let $\pi_{0} \hat{M}_{i}(f)$ denote the set of connected components of $\hat{M}_{i}(f)$. The invariant $\mu_{2}$ is a function: $\mu_{2}: \pi_{0} \hat{M}_{2}(f) \rightarrow\{0,1\}$ where $\mu_{2}(\alpha)=0$ iff the covering $P_{2}^{-1}(\alpha) \rightarrow \alpha$ is trivial. In view of Corollary 2 , the following remark might be useful:

Proposition. Let $M, N$ be manifolds of dimensions 2 and 3, $M$ closed, $\partial N=\varnothing . M$ and $N$ are supposed orientable and

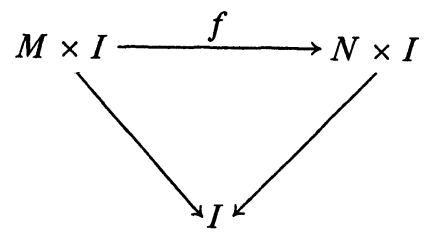

is a generic regular homotopy. Then $\mu_{2}(f)=0$ if and only if $\hat{M}_{2}(f)$ is orientable.

3. We will define now invariants $v_{3}, v_{4}, \ldots$ which are the "good" generalization of $\mu_{2}$. Only $\nu_{3}$ is need here, but it is perhaps more enlightening to define them all.

We consider the inverse system:

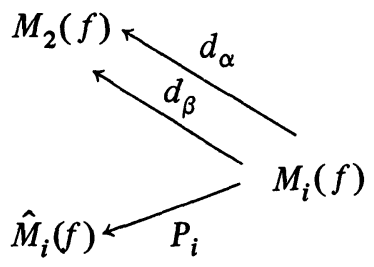

where $d_{\alpha}, d_{\beta} \cdots$ are the $i(i-1)$ natural ways of an oriented $i$-tuple point to degenerate into an oriented double point. 
The "limit" of this system lies in $M_{2} \times M_{i} \times \hat{M}_{i}$, and its projection in $M_{2} \times$ $\hat{M}_{i}$ is denoted by $S_{2, i}$. One has a natural projection, which is a covering map $S_{2, i} \stackrel{Q_{i}}{\longrightarrow} \hat{M}_{i}$ and if $\hat{M}_{i} \ni x=\left\{x_{1}, \ldots, x_{i}\right\}$ (the set of distinct points $x_{1}, \ldots$, $\left.x_{i}\right)$ then $Q_{i}^{-1}(x)=x \times x-\operatorname{diag} x$.

The structural group of the fibration $Q_{i}$ is reduced; instead of being $S(i(i-1))$ it is $S(i)=\operatorname{Perm}\left\{x_{1}, \ldots, x_{i}\right\}$.

On $S_{2, i}$ we introduce the equivalence relation $\sim\left(\sim_{i}\right)$ which is, by definition, the smallest equivalence relation with the followign properties:

(a) $\left(x^{\prime}, x^{\prime \prime}\right) \sim\left(y^{\prime}, y^{\prime \prime}\right) \Rightarrow\left(x^{\prime \prime}, x^{\prime}\right) \sim\left(y^{\prime \prime}, y^{\prime}\right)$.

(b) If $\left(x^{\prime}, x^{\prime \prime}\right),\left(y^{\prime}, y^{\prime \prime}\right) \in S_{2, i} \subset M_{2} \times \hat{M}_{i} \rightarrow M_{2}$

have their images in the same connected components of $M_{2}$, they are equivalent.

(c) Let $x \in \hat{M}_{i}$ and consider $g \in S(i)$, a circular permutation of length $i$, acting on the fiber $Q_{i}^{-1}(x)$. If

$$
\left(x_{r}, x_{j}\right)=y \sim g y \sim g^{2} y \sim \cdots \sim g^{i-2} y=\left(x_{j}, x_{k}\right),
$$

then $\left(x_{r}, x_{k}\right) \sim\left(x_{r}, x_{j}\right)$.

If $i=3$, property (c) means just that

$$
\left(x_{r}, x_{j}\right) \sim\left(x_{j}, x_{k}\right) \Rightarrow\left(x_{r}, x_{j}\right) \sim\left(x_{r}, x_{k}\right) .
$$

(Note that in (c) everything is in one fiber; in (a), (b) this is not necessarily so.)

We shall define $\nu_{i}: \pi_{0} \hat{M}_{i} \rightarrow\{0,1, \ldots\}(i \geqslant 3)$, as follows:

If $x \in \alpha \in \pi_{0} \hat{M}_{i}$, we consider the number of distinct subsets $E \subset Q_{i}^{-1}(x)$ such that:

(a) $E$ has $i$ elements.

(b) There exists $y=\left(x_{j}, x_{k}\right) \in Q_{i}^{-1}(x)$ and $g \in S(i)$, circular permutation of length $i$, such that $E=\left\{y, g, y, \ldots, g^{i-1} y\right\}$.

(c) $y \sim g y \sim \cdots \sim g^{i-1} y$.

[Two sets $E, E^{\prime}$ obtained one from the other by $\left(x_{j}, x_{k}\right) \rightarrow\left(x_{k}, x_{j}\right)$ will not be regarded as distinct.]

The number of distinct $E$ 's is, by definition, $\nu_{i}(\alpha)$. (This number is independent of $x \in \alpha$.)

FINAL REMARKS. (1) The notations of [2] are slightly different from the notations used here. In [2], $M_{i}$ becomes $M_{i}$ and $S_{2, i}$ becomes $S_{2, i} \cdot M_{i}$ from [2] is the set of i-tuple points in $X \times S^{i-1} X$. Looking at the i-tuple points at the level $X \times S^{i-1} X$ means, exactly, blowing up the singularities of $M^{i}(f) \subset X$ in order to get a smooth manifold (i.e., what one gets at the $X \times S^{i-1} X$ level is the "resolution of singularities" for $\left.M^{i}(f)\right)$.

(2) We have assumed here that $\operatorname{dim} X<\operatorname{dim} Y$ (or $\operatorname{dim} X=\operatorname{dim} Y, \partial Y=$ $\varnothing, X$ compact bounded, and $f \mid \partial X$ generic). Otherwise, the preceding theory is to be replaced by the following remark: A (connected) covering map $X \stackrel{f}{\rightarrow} Y$ 
can be lifted to $Y \times R$ if and only if it is infinitely cyclic.

(3) The invariant $\mu_{2}$ (for the case $\operatorname{dim} X=\operatorname{dim} Y=3, \ldots$ ) turns out to be deeply connected to the handle-body structure of $\Sigma_{3} \times I$ [3]

\section{BIBLIOGRAPHY}

1. M. A. Kervaire and J. W. Milnor, Groups of homotopy spheres. I, Ann. of Math. (2) 77 (1963), 504-537. MR 26 \#5584.

2. V. Poénaru, Homotopie réguliere et isotopie (to appear).

3. - Sur la structure des sphères d'homotopie lisses en dimension 3, I, II (to appear).

DÉPARTMENT OF MATHEMATICS, FACULTE DES SCIENCES D’ORSAY, 91-ORSAY, FRANCE 